\title{
Highly efficient photoinduced phase transition in an organic radical crystal via two-photon absorption process
}

\author{
Toyoki Kon, ${ }^{1}$ Seiji Oguri, ${ }^{1}$ Ikufumi Katayama, ${ }^{2}$ Tetsuya Kodaira, ${ }^{3}$ and Jun Takeda ${ }^{1}$ \\ ${ }^{1}$ Department of Physics, Graduate School of Engineering, Yokohama National University, 79-5 Tokiwadai, Hodagaya-ku, \\ Yokohama 240-8501, Japan \\ ${ }^{2}$ Interdisciplinary Research Center, Yokohama National University, 79-5 Tokiwadai, Hodogaya-ku, Yokohama 240-8501, Japan \\ ${ }^{3}$ Nanoarchitectonics Research Center, National Institute of Advanced Industrial Science and Technology (AIST), 1-1 Higashi, \\ Tukuba 305-8565, Japan
}

(Received 17 November 2008; published 7 January 2009)

\begin{abstract}
We have studied excitation energy dependence of the conversion yield of two-photon-mediated phase transition in organic radical 1,3,5-trithia-2,4,6-triazapentalenyl (TTTA) crystal. Even under photoexcitation far below the lowest charge-transfer absorption edge, the photoinduced phase transition takes place from diamagnetic low-temperature phase to paramagnetic high-temperature phase with threshold excitation density. By comparing the excitation energy dependence of the conversion yield with the two-photon absorption spectrum, we found that the conversion is resonantly enhanced by two-photon absorption process. Because of the strong three-dimensional interaction between the excited states of TTTA molecules, the homogeneous two-photon excitation can induce the phase transition with high efficiency.
\end{abstract}

DOI: 10.1103/PhysRevB.79.035106

PACS number(s): 64.70.kt, 75.50.Xx

Photoinduced (PI) phase transition (PIPT) has been one of the major subjects of recent researches in solid-state physics because it involves nonlinear dynamics associated with the cooperative interaction between excited states, nonequilibrium nature, and possibility to induce a new phase which is impossible to access via the thermal phase transition. ${ }^{1}$ Various organic materials such as spin-crossover complexes, ${ }^{2-4}$ organic ionic crystals, ${ }^{5}$ and organic polymer crystals ${ }^{6}$ are found to show the PIPT, and the extensive studies of these materials including ultrafast time-resolved measurements reveal the important features such as existence of incubation time and threshold density to drive the PIPT. ${ }^{1,3}$ However, as far as we know, all of the PIPT studies has been performed using one-photon process, which causes inhomogeneity due to finite penetration depth of the excitation light; diffusion of the excited states and excess heat generated by the excitation light obscure real features of the PIPT dynamics. ${ }^{5}$ Furthermore, the inhomogeneous stress caused by the difference of the unit-cell volume between ground states and excited states might also affect the transition. In order to elucidate the PIPT dynamics without such inhomogeneities, exploration of new materials that show the PIPT by two-photon absorption process is highly desired, where we can reasonably assume that the excitation is homogeneous.

Strongly correlated organic radical 1,3,5-trithia-2,4,6triazapentalenyl (TTTA) crystal is one of the promising candidates for such studies. TTTA molecule consists of eight atoms: three sulfur, three nitrogen, and two carbon atoms. In Fig. 1, we show the chemical formula and the crystal structure of two phases in TTTA crystal. TTTA has a large magnetic bistability around room temperature, ${ }^{7}$ and shows the PIPT from a diamagnetic low-temperature (LT) to paramagnetic high-temperature (HT) phase inside the hysteresis loop. ${ }^{8,9}$ The HT phase is a Mott insulator due to the strong correlation between the unpaired electrons in TTTA molecules, and these unpaired electrons form dimers to become diamagnetic LT phase. ${ }^{10}$ Both phases have larger band gaps of about $1.7 \mathrm{eV}$ than many of the other materials showing PIPT (typically $0.6 \mathrm{eV}$ ). Therefore, as far as we know, TTTA is a unique and suitable material for the study of two-photoninduced phase transition, where the diffusion effects and excitation inhomogeneity can be neglected and efficient PIPT can be expected.

In this work, we measure the photoinduced Raman spectra under various excitation conditions and demonstrate that the conversion yield of the PIPT in TTTA is resonantly enhanced by the two-photon absorption. The resonant behavior is examined by the comparison with the excitation spectrum of two-photon luminescence intensity from self-trapped excitons (STEs). ${ }^{11}$ Because the two-photon absorption process can dramatically reduce the thermal diffusion, stress from the spatial inhomogeneity, and so on, it offers clear and useful insight into the dynamics of PIPT. In addition, since the twophoton process can excite the whole volume of the crystal, the conversion yield and its efficiency of the phase transition might become much larger than that of the one-photon excitation. $^{12}$

(a)

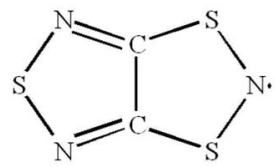

(b)

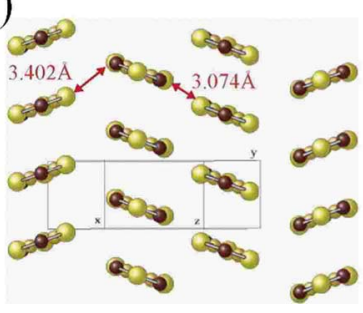

(c)

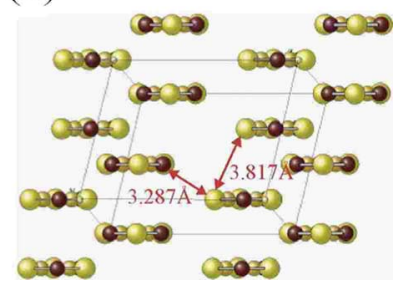

FIG. 1. (Color online) (a) Chemical formula of TTTA molecule and the crystal structure of the TTTA crystals in (b) HT phase and (c) LT phase. 


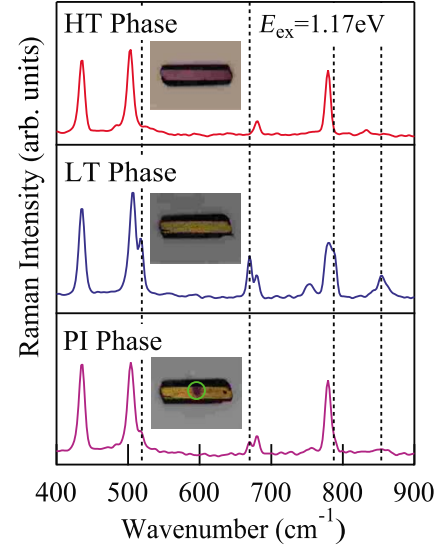

FIG. 2. (Color online) Raman spectra and pictures of the grown crystals in HT paramagnetic phase, LT diamagnetic phase, and PI phase excited at $1.17 \mathrm{eV}$ with $3.6 \times 10^{18}$ photons $/ \mathrm{cm}^{2}$ at room temperature. Dotted lines indicate characteristic peaks of the LT phase whose intensities decrease in the HT and PI phases. A small circle in the picture of PI phase corresponds to the excitation spot.

TTTA crystals were grown by the method previously reported. ${ }^{7,13}$ The grown crystals were in HT phase with the typical size of $1 \times 0.3 \times 0.1 \mathrm{~mm}^{3}$. The LT phase can be obtained by the cooling of the crystals to $77 \mathrm{~K}$ by liquid nitrogen. TTTA crystals were excited by the laser light from an optical parametric oscillator (OPO) pumped with an $\mathrm{Nd}$ doped yttrium aluminum garnet (YAG) laser. The output photon energy of the laser ranges from 0.56 to $1.38 \mathrm{eV}$ with the pulse duration and the repetition rate of $5 \mathrm{~ns}$ and $10 \mathrm{~Hz}$. After each one-pulse excitation on the LT phase crystal at a given photon density, we measured the Raman spectra by the Fourier-Raman spectrometer with a cw-Nd:YAG laser (1.17 eV). ${ }^{9}$ Because the excitation intensity of the $\mathrm{cw}$-YAG laser is weak enough and the wavelength is much longer than the lowest absorption edge of both the HT and LT phases, we can measure the photoinduced Raman signals from a bulk of the sample. In addition, we estimated the two-photon absorption spectrum from the two-photon luminescence intensity excited by the OPO laser system with sufficiently low power which makes no PIPT. The luminescence intensity was measured by a single grating spectrometer with a charge-coupled device (CCD) detector cooled at $223 \mathrm{~K}$. The wavelengths and sensitivities are corrected by the $\mathrm{Hg}$ emission line and the standard blackbody light source. All the experiments were performed in room temperature.

Figure 2 shows Raman spectra and pictures of TTTA crystals in HT paramagnetic, LT diamagnetic, and PI phases. The Raman spectrum in the PI phase is obtained under singleshot excitation on LT phase crystal with $E_{\mathrm{ex}}=1.17 \mathrm{eV}$ whose energy is much below the lowest charge-transfer absorption edge $(1.4 \mathrm{eV})$. Comparing the Raman spectrum of HT phase with that of LT phase, we can see that some Raman peaks (for example, 515, 670, and $780 \mathrm{~cm}^{-1}$ ) split into two peaks and another peak $\left(850 \mathrm{~cm}^{-1}\right)$ appears in LT phase, which are related to the zone-boundary folding due to the doubled unit cell and the dimerization of the neighboring TTTA molecules.

It is important that the PI phase has the different Raman
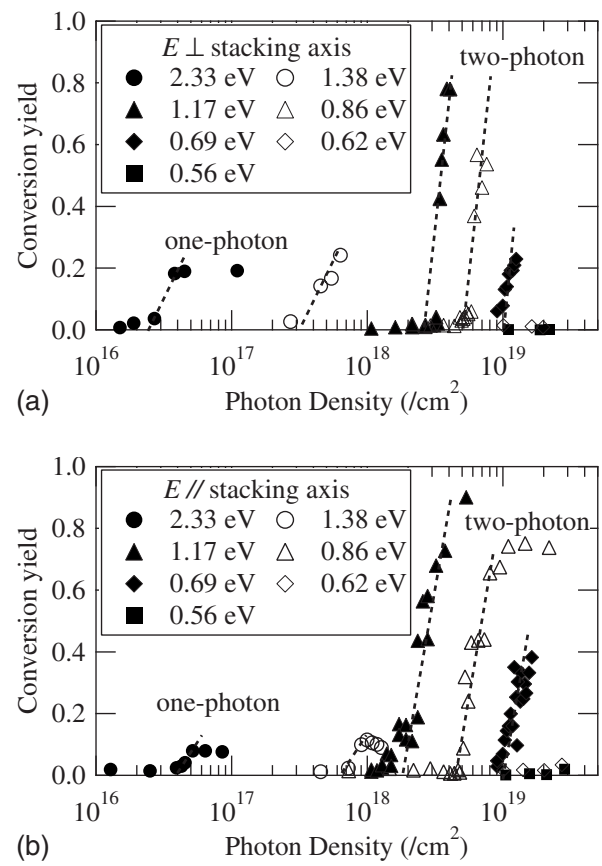

FIG. 3. Excitation photon density dependence of the conversion yield estimated from the Raman spectra under the excitation at various excitation energies with the polarization (a) perpendicular and (b) parallel to the stacking axis of TTTA crystals at room temperature. Dotted lines are the guide for the eyes. Note that the lowest absorption edge of TTTA crystals in LT phase is around $1.4 \mathrm{eV}$. Since the two-photon absorption coefficient of TTTA is unknown, the horizontal axis is in the unit of photon density per unit area of the crystal surface, not per unit volume as in the Ref. 9.

features from LT phase even via the excitation in the transparent region. In addition, the color of the excited spot changes to purple which indicates the transition to HT phase. This PI phase is stable for at least several days at room temperature. To estimate the conversion yield of this transition, we decompose the Raman spectrum of the PI phase into a sum of the spectra of HT and LT phases. ${ }^{9}$ In Fig. 3, we show the estimated conversion yield from the LT to the HT phase as a function of the excitation photon density. The plotted data are obtained under the excitation of the laser light $(0.56,0.62,0.69,0.86,1.17$, and $1.38 \mathrm{eV})$ with the parallel $\left(E_{\|}\right)$and perpendicular $\left(E_{\perp}\right)$ polarizations to the stacking direction of TTTA molecules. For a comparison, the conversion yields by the one-photon process $(2.33 \mathrm{eV})$ are also shown. ${ }^{12}$

As is guided by the dotted lines in this figure, the conversion yield increases above a certain threshold excitation density. This kind of threshold behavior is one of the characteristics of the PIPT and suggests existence of the cooperative interaction between the excited states. We can also see that the threshold photon densities under 1.17, 0.86 , and $0.69 \mathrm{eV}$ excitations are about 2 orders of magnitude larger than that under $2.33 \mathrm{eV}$ excitation, and the conversion yield ranges from 0.4 to unity when the excitation light is in the transparent energy region. In addition, the steepness of the dotted lines in Fig. 3 under 1.17, 0.86, and $0.69 \mathrm{eV}$ excitations is much larger than that under $2.33 \mathrm{eV}$, namely, the one-photon excitation. Therefore, nonlinear optical process, such as two- 


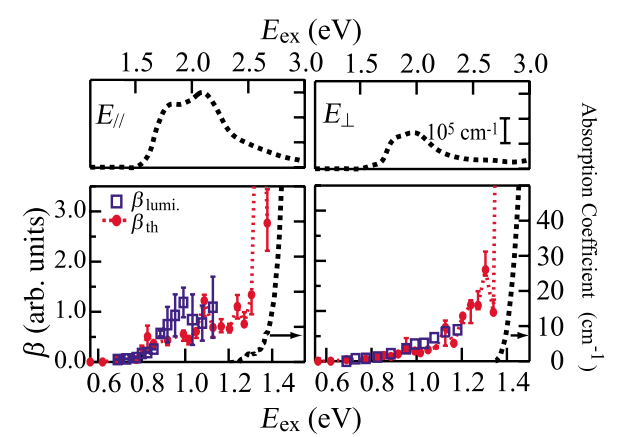

FIG. 4. (Color online) Excitation photon energy dependence of the two-photon absorption coefficient estimated from the twophoton luminescence intensity $\left(\beta_{\text {lumi }}\right)$ and from the threshold excitation density of the PIPT as mentioned in the text $\left(\beta_{\mathrm{th}}\right)$. The dotted lines are the one-photon absorption spectra of TTTA crystals in the LT phase.

photon absorption process, should induce the PIPT under $1.17,0.86$, and $0.69 \mathrm{eV}$ excitations. Under the $1.38 \mathrm{eV}$ excitation (open circles), the conversion yield and the steepness of the threshold behavior are the same as those under the one-photon process $(2.33 \mathrm{eV})$, suggesting that the excitation might be in one-photon regime since the energy is close to the lower tail of the lowest charge-transfer absorption. On the other hand, the 0.56 and $0.62 \mathrm{eV}$ excitations cannot induce the phase transition even at high photon density, where the energy of two photons is far below the lowest absorption band. Since the $0.69 \mathrm{eV}$ excitation is close to the lowest edge of the two-photon resonance, the conversion yield may not reach to unity but is limited to around 0.4 .

To confirm that the phase transition is really induced by the two-photon absorption process, we compare the energy dependence of the conversion yield with the two-photon absorption spectrum of the LT phase. Assuming that the threshold nucleus density $n_{\text {th }}$ to drive the PIPT is independent of the excitation energy $E_{\mathrm{ex}}$ and that the phase transition occurs due to two-photon absorption process, the threshold excitation photon density $I_{\mathrm{th}}\left(E_{\mathrm{ex}}\right)$ might be related to $n_{\mathrm{th}}$ as

$$
n_{\mathrm{th}} \propto \beta_{\mathrm{th}}\left(E_{\mathrm{ex}}\right) I_{\mathrm{th}}^{2}\left(E_{\mathrm{ex}}\right) .
$$

Here, $\beta_{\mathrm{th}}\left(E_{\mathrm{ex}}\right)$ is the conversion yield efficiency as a function of the excitation photon energy, which should be proportional to the two-photon absorption coefficient. On the other hand, the two-photon absorption coefficient $\beta_{\text {lumi }}\left(E_{\mathrm{ex}}\right)$ can be estimated from the two-photon luminescence intensity $I_{\text {lumi }}\left(E_{\text {ex }}\right)$ when the excitation intensity is small enough to ensure that the higher-order nonlinear processes can be neglected. ${ }^{12}$ The two-photon absorption coefficient is proportional to the two-photon luminescence intensity,

$$
\beta_{\text {lumi }}\left(E_{\text {ex }}\right) \propto I_{\text {lumi }}\left(E_{\text {ex }}\right) .
$$

In Fig. 4, we plot the two-photon absorption coefficient $\beta_{\text {lumi }}\left(E_{\mathrm{ex}}\right)$ estimated from the two-photon luminescence intensity under the low-power excitation condition and the conversion efficiency $\beta_{\mathrm{th}}\left(E_{\mathrm{ex}}\right)$ estimated from the threshold excitation photon density of the PIPT as a function of excitation energy $E_{\mathrm{ex}}$. As seen in this figure, $\beta_{\mathrm{th}}\left(E_{\mathrm{ex}}\right)$ is in quite good agreement with the two-photon absorption spectrum $\beta_{\text {lumi }}\left(E_{\text {ex }}\right)$, strongly suggesting that the PIPT under the excitation below the charge-transfer absorption edge of LT phase is mainly due to the two-photon absorption process. In addition, no PIPT takes place under the excitation below $0.65 \mathrm{eV}$, whose energy is below the two-photon resonance. The good coincidence of $\beta_{\text {lumi }}\left(E_{\mathrm{ex}}\right)$ and $\beta_{\mathrm{th}}\left(E_{\mathrm{ex}}\right)$ also indicates that the STEs play important roles for PIPT. ${ }^{1}$ This is an observation of the PIPT induced by the two-photon absorption process.

Now, let us consider the difference of PIPT behaviors between the two-photon and the one-photon processes. The main difference originates from the difference of the penetration depth of the excitation light. The estimated penetration depth of the excitation light for one-photon process is on the order of few nanometers to few hundred nanometers for the photon energy from 1.4 to $4 \mathrm{eV},{ }^{14}$ which is at least 3 orders of magnitude smaller than the thickness of the crystal. However, the PIPT under one-photon process still occurs having about $10 \%-20 \%$ conversion yield (2.33 eV excitation; see Fig. 3) of the LT phase crystal, probably because of the excited-state diffusion or the heat conduction from the surface into interior regions. Therefore, it is very difficult to extract the real characteristics of the PIPT beyond these ambiguities under the one-photon process.

On the other hand, these ambiguities can be neglected in the two-photon regime since the energy of the excitation light is in the transparent region and the excitation can be regarded as uniform. Indeed, as shown in Fig. 4, the absorption coefficient from 0.56 to $1.3 \mathrm{eV}$ is smaller than $1 \mathrm{~cm}^{-1}$, suggesting that the penetration depth in this energy region is much larger than the crystal thickness of about $100 \mu \mathrm{m}$. In the following, we try to estimate the real threshold density per unit volume to drive the PIPT under the two-photon process in order to compare it with that under the one-photon process.

During the two-photon experiment, we measured the output power of the excitation light transmitted through the TTTA crystal. The ratio of the transmitted power to the incident power is almost constant, which is independent of the excitation power. The actual absorption due to the twophoton process estimated is at least less than $1 \%$ of the input power under the experimental accuracy, which ensures the homogeneous excitation condition. Therefore the threshold photon density under two-photon process is about $10^{16} \mathrm{~cm}^{-2}$ and is comparable to that under the one-photon process $(2.33$ $\mathrm{eV}$ ) as shown in Fig. 3. Furthermore, the estimated excited volume of the TTTA crystal under two-photon process ( $\sim 100 \mu \mathrm{m})$ is much larger than that under one-photon process $(\sim 100 \mathrm{~nm})$. This fact indicates that the real threshold density of the excited states per unit volume to drive the PIPT in two-photon regime must be much lower than that in one-photon regime, implying that the two-photon-induced phase transition is very efficient.

In the PIPT, cooperative interaction between the excited states is important for driving the PIPT. As is schematically shown in Fig. 5(a), the one-photon process can only excite near the surface of the crystal. Then the interaction between the excited states is limited in the surface region, namely, two dimensions. The phase conversion in the depth originates from the thermal diffusion or the inhomogeneous 
(a) one photon abs.
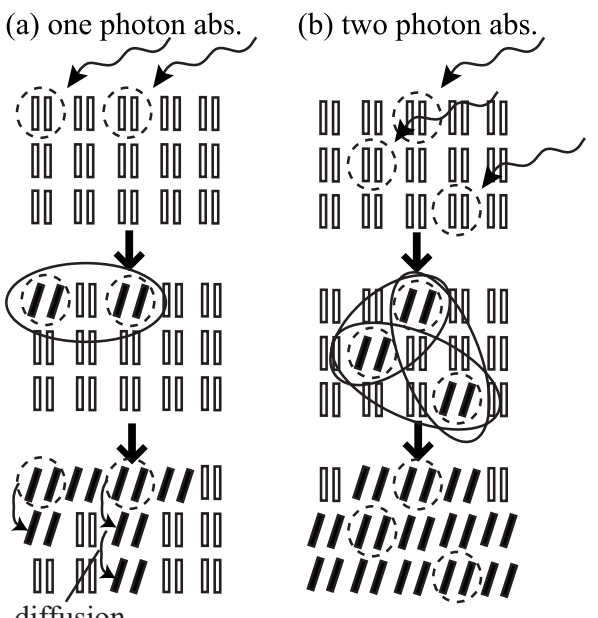

FIG. 5. Schematic viewgraph of the cooperative interaction between the excited states that realizes PIPT. In (a) the one-photon process, the interaction is limited in two dimensions at the surface. The phase conversion inside the crystal is due to the thermal diffusion or stress which masks the real PIPT behavior. In contrast, (b) two-photon process offers optically induced three-dimensional interaction as depicted by the solid circles.

stress, which masks the real PIPT behavior. On the other hand, by using the two-photon process, the excitation is almost homogeneous and the cooperative interaction takes place three dimensionally as shown in Fig. 5(b). Strong three-dimensional interaction in TTTA is theoretically predicted as a large intermolecular transfer integral of N-S or $\mathrm{S}-\mathrm{S}$ bonding. ${ }^{10,14}$ Then the stabilization of the excited states under two-photon process should be much faster than that under one-photon process, leading to the conversion yield with high efficiency and sharp steepness as shown in Fig. 3.

In conclusion, the TTTA crystals undergo the PIPT even under the excitation of the transparent region via the twophoton absorption process. The estimated energy dependence of the PIPT efficiency is consistent with the two-photon absorption spectrum estimated from the two-photon luminescence due to STEs. The efficiency shows resonant enhancement with the two-photon absorption edge, and the conversion yield reaches to 0.9 , which is much larger than that under the one-photon absorption. Since the unpaired electrons in TTTA crystals are strongly correlated, the cooperative interaction between the photoexcited states is extremely large to realize the PIPT with high efficiency. Because the two-photon absorption can be regarded as a uniform excitation, the dynamics of the PIPT can easily be studied without ambiguity due to inhomogeneity. Such studies, therefore, give clear and fruitful insights into the physical understanding of the PIPT.

This work was partly supported by the Special Coordination Funds for Promoting Science and Technology from Japan Science and Technology Agency (JST) and by the Grantin-Aids for Scientific Research (A) from the Japan Society for the Promotion of Science (JSPS) and for Scientific Research on Priority Areas "Development of New Quantum Simulator and Quantum Design" from the Ministry of Education, Culture, Sports, Science and Technology (MEXT). We thank K. Ohno in Yokohama National University for his fruitful discussion.
${ }^{1}$ Photoinduced Phase Transitions, edited by K. Nasu (World Scientific Publishing, Singapore, 2004).

${ }^{2}$ T. Tayagaki and K. Tanaka, Phys. Rev. Lett. 86, 2886 (2001).

${ }^{3}$ Y. Ogawa, S. Koshihara, K. Koshino, T. Ogawa, C. Urano, and H. Takagi, Phys. Rev. Lett. 84, 3181 (2000).

${ }^{4}$ S. Marcén, L. Lecren, L. Capes, H. Goodwin, and J. F. Létard, Chem. Phys. Lett. 358, 87 (2002).

${ }^{5}$ H. Okamoto, Y. Ishige, S. Tanaka, H. Kishida, S. Iwai, and Y. Tokura, Phys. Rev. B 70, 165202 (2004).

${ }^{6}$ S. Koshihara, Y. Tokura, K. Takeda, and T. Koda, Phys. Rev. Lett. 68, 1148 (1992).

${ }^{7}$ W. Fujita and K. Awaga, Science 286, 261 (1999).

${ }^{8}$ H. Matsuzaki, W. Fujita, K. Awaga, and H. Okamoto, Phys. Rev.
Lett. 91, 017403 (2003).

${ }^{9}$ J. Takeda, M. Imae, O. Hanado, S. Kurita, M. Furuya, K. Ohno, and T. Kodaira, Chem. Phys. Lett. 378, 456 (2003).

${ }^{10}$ K. Ohno, Y. Noguchi, T. Yokoi, S. Ishii, J. Takeda, and M. Furuya, ChemPhysChem 7, 1820 (2006).

${ }^{11}$ Y. Takahashi, T. Suemoto, S. Oguri, and J. Takeda, Phys. Rev. B 74, 193104 (2006).

${ }^{12}$ T. Kon, S. Oguri, and J. Takeda, J. Lumin. 128, 774 (2008).

${ }^{13} \mathrm{G}$. Wolmershäuser and R. Johann, Angew. Chem., Int. Ed. Engl. 28, 920 (1989).

${ }^{14}$ W. Fujita, K. Awaga, H. Matsuzaki, and H. Okamoto, Phys. Rev. B 65, 064434 (2002). 